\title{
The Use of Magnets in Orthodontics
}

\author{
${ }^{1}$ Narendra Sharma, ${ }^{2}$ Sunita Shrivastav, ${ }^{3} \mathrm{RH}$ Kamble, ${ }^{4}$ Preethi Sharma
}

\begin{abstract}
Magnets have generated great interest within dentistry. They have been used for various applications in orthodontics and prosthodontics. Earlier use of magnets was limited due to the unavailability of small size magnets, but after the introduction of rare earth magnets and their availability in smaller sizes, their use has increased considerably. The force they deliver can be directed, and they can exert their force through mucosa and bone as well as within the mouth. In orthodontics, they are used for intrusion of teeth, tooth movement along archwires, expansion, retention, in functional appliances and in the treatment of impacted teeth. There are various types of magnets used in the field of orthodontics with their advantages and disadvantages, along with their biological safety which has been discussed in this article. This article reviews various uses of magnets in the field of orthodontics.
\end{abstract}

Keywords: Magnets, Malocclusion, Orthodontics.

How to cite this article: Sharma N, Shrivastav S, Kamble $\mathrm{RH}$, Sharma P. The Use of Magnets in Orthodontics. World J Dent 2015;6(1):45-48.

Source of support: Nil

Conflict of interest: None

\section{INTRODUCTION}

We live in an environment of magnetic fields, both natural and artificial. Evolution and normal biologic process may well be magnetic field dependent. We are always exposed to the magnetic fields of significant intensity at home, in our automobiles, in wrist-watches, and when walking under high power electric lines. ${ }^{1}$ Magnets have been used in dentistry for many years, most commonly to aid the retention of dentures and overdentures. ${ }^{2-4}$ In orthodontics, they have been used in both research and clinical practice, particularly in the treatment of unerupted teeth, ${ }^{5,6}$ for tooth movement along archwires, ${ }^{7}$ expansion, fixed retention, ${ }^{8}$ in the correction of anterior

\footnotetext{
${ }^{1}$ Associate Professor, ${ }^{2}$ Professor and Head, ${ }^{3}$ Professor

${ }^{4}$ Senior Resident

${ }^{1-3}$ Department of Orthodontics, Sharad Pawar Dental College Wardha, Maharashtra, India

${ }^{4}$ Department of Oral Pathology, Sharad Pawar Dental College Wardha, Maharashtra, India
}

Corresponding Author: Narendra Sharma, Associate Professor Department of Orthodontics, Sharad Pawar Dental College Wardha, Maharashtra, India, Phone: 9326390639, e-mail: sharmanarendra047@gmail.com open bite and in functional appliances. Magnets are said to have significant advantages over other materials used to move teeth, such as elastic chain or push-coil, as they are able to produce a measured force continuously over long periods of time for various kinds of tooth movement. They can be made to attract or repel and the force they deliver can be directed, and can exert their force through mucosa and bone.

\section{Biological Safety}

It is important to ensure that magnets used intraorally for clinical use should not produce any side-effects at a local or systemic level. A full evaluation must include three levels of testing as follows:

- Level 1: In vitro testing in order to establish the toxic, allergic or carcinogenic nature of the material.

- Level 2: In use testing on animals.

- Level 3: Clinical trials. Magnets used in orthodontics produce static magnetic fields. Biological testing of magnets containing rare earth elements has evaluated the effects of both the static magnetic field, and possible toxic effects of the materials or their corrosion products. Lars Bondemark and Jure Kurol compared in vitro the cytotoxic effects of uncoated and parylene coated rare earth magnet by using two methods as follows: ${ }^{9}$

1. Millipore filter method

2. Extraction method

\section{TYPES OF MAGNETIC MATERIALS}

In various dental applications, the following materials have been used:

- Platinum-Cobalt (Pt-Co)

- Aluminum-nickel-cobalt (Al-Ni-Co)

- Ferrite

- Chromium-cobalt-iron (Cr-Co-Fe)

- Samarium-cobalt (Sm-Co)

- Neodymium-iron-boron (Nd-Fe-B)

\section{CLASSIFICATION OF MAGNETS}

There has been no definite classification of magnets given in the literature. Hence, we have tried to combine different types mentioned in various articles, and an effort has been made to develop a simplified classification system for magnets. 
1. Based on alloys used

- Those containing cobalt, e.g. Alnico, Alnico V, Co$\mathrm{Pt}, \mathrm{Co}_{5} \mathrm{Sm}$

- Those not containing cobalt, e.g. Nd-Fe-B, samarium iron nitride

2. Based on ability to retain magnetic properties (intrinsic coercivity or hardness)

- Soft (easy to magnetize or demagnetize) (less permanent), e.g. Pd-Co-Ni alloy, Pd-Co alloy, Pd-CoCr alloy, Pd, Co-Pt alloy, magnetic stainless steels, Permendur (alloy of Fe-Co), Cr-Molybdenum alloy.

- Hard (retain magnetism permanently). For example, Alnico alloys, $\mathrm{Co}-\mathrm{Pt}, \mathrm{Co}_{5} \mathrm{Sm}, \mathrm{Nd}-\mathrm{Fe}-\mathrm{B}$.

3. Based on surface coating (materials may be stainless steel, titanium or palladium)

- Coated

- Uncoated

4. Based on the type of magnetism

- Repulsion

- Attraction

5. Based on type of magnetic field

- Open field

- Closed field

- Rectangular closed-field sandwich design

- Circular closed-field sandwich design

6. Based on number of magnets in the system

- Single

- Paired

7. Based on the arrangement of the poles

- Reversed poles

- Nonreversed poles

\section{ATTRACTING MAGNETS}

1. Increased activation may be built into the initial construction bite for appliances using attracting magnets.

2. The attracting magnetic force pulls the appliances together and encouraging the patients to occluded actively and consistently in a forward position.

3. Clark has used two materials, $\mathrm{SmCO}_{5}$ and Neodymium boron, to test the clinical response to magnetic twin blocks:

- Neodymium boron applies a higher magnetic force from smaller magnets but is more likely to corrode if not adequately protected from abrasion.

4. Attracting magnets were used clinically in different situations as follows:

- Class II div 1 malocclusion with a large over jet.

- Mild residual class II buccal segment relationship.

- Mild class II Div 1 malocclusion with an over jet of $7 \mathrm{~mm}$.

- Unilateral class II adult patient with temporomandibular joint pain.
- Skeletal class III malocclusion with persistent cross bite.

- Correction of facial asymmetry.

\section{REPELLING MAGNETS}

1. Repelling magnets may be used in twin blocks with a lesser mechanical activation built into the inclined planes.

2. The repelling magnets were intended to induce additional forward mandibular posture without the need for reactivation of blocks.

3. Whether attracting or repelling magnets are used, reactivation of block by addition of acrylic to the inclined planes deactivates the magnets.

4. So, screws are included in the appliance design for magnetic twin blocks to achieve continuous reactivation of magnetic force.

5. Attracting magnets are indicated in cases in which the patient does not or cannot make the muscular effort to posture consistently to the corrected occlusion.

6. Magnets should be used only where speed of treatment is an important consideration, or where the response to nonmagnetic appliances is limited.

\section{Simple Tooth Movement without Archwires}

Muller ${ }^{10}$ suggested the use of small magnets (approximately $531 \mathrm{~mm}$ ) to deliver light continuous forces to close diastemas without archwires as they deliver predictable, constant low forces. The magnets were bonded to the labial aspect of the teeth. The force delivered was determined by the distance apart the teeth were and, therefore, the size of magnet bonded. Muller suggests that rotations and angulation problems can also be corrected with this technique. The magnets produce a light continuous force that increases as the teeth get nearer is the reason the teeth move quickly.

\section{Expansion}

Intramaxillary expansion and orthopedic movement of the palatal shelves have been used in orthodontics for many years. Vardimon et al reported on a study that looked into the effects of using samarium-cobalt magnets to provide the expansion force on monkeys. ${ }^{11}$ This demonstrated that magnetic expansion does produce controlled forces over a predicted range and time. The expansion is slow compared with rapid maxillary expansion and, consequently, there are fewer tendencies for the mid-palatal suture to fracture. In addition, as the forces can be made to be more physiological it avoids the complications of the rotations of the maxilla seen in the high force appliances, such as RME. 


\section{Molar Distalization with Magnets}

One of the effective methods to resolve a class II malocclusion is the distal movement of upper molars to establish a class I relationship. The premolars and canines are subsequently moved back to class I positions and, finally, the incisors retracted. Repelling magnets can provide continuous force need to establish a class I molar relationship in the early mixed dentition, Gianelly et al used intra-arch repelling magnets to distalize the maxillary molars. ${ }^{12}$

\section{Retainers}

Despite the success of fixed retainers to stabilize anterior spacing which are often used in orthodontics, they have a number of undesirable characteristics. They restrict access to the gingival tissues, leading to poor oral hygiene, and they often fracture because the individual teeth move independently and excessive strain on the retainer. Micromagnetic retainers have been suggested by to retain central incisors that have been brought together to close a median diastema. After tooth movement, small neodymium-ironboron magnets are bonded with a light-cured low viscosity resin on the mesiopalatal aspect of the teeth separated during bonding by an acetate finishing strip to ensure the two magnets are not fused together. Directly bonded magnets have a number of advantages over other types of retainer.

\section{Functional Appliances}

Magnets have been used for the correction of class II and III malocclusions. Vardimon et al developed the functional orthopedic device (FOMA II and III), which has shown positive treatment effects in monkeys. ${ }^{13}$ In the case of FOMA II, upper and lower attracting neodymium-iron-boron magnets maintain the mandible in an advanced sagittal position. The objectives of the study were to develop an appliance capable of leaving the mandible in the advanced position and to establish a skeletal response. The first clinical experience with a magnetic activator device (MAD) for the correction of a class II division 1 malocclusion and another device for class III cases has recently been described. ${ }^{14}$ Several types have been designed to deal with differing clinical problems, e.g. lateral displacement (MAD I), class II malocclusions (MAD II), class III (MAD III), and open bite cases (MAD IV). Chate describes the propellant unilateral magnetic appliance (PUMA) in the treatment of hemifacial microsomia. ${ }^{15}$ This appliance uses samarium-cobalt magnets. Moss has described the use of the twin block appliance. ${ }^{16}$

\section{Magnets for Tooth Movement}

The first reported use of magnetic force to move teeth was in 1977 when Kawata and Takeda described a technique of using magnetic brackets of $\mathrm{Co}-\mathrm{Cr}-\mathrm{Fe}$ alloy, bonded to the upper anterior and the lower anterior teeth for interdental space closure. ${ }^{17}$ The 'retracting and pushing' force of the maxillary brackets was reported to be 25 to $30 \mathrm{gm}$ and that in the mandibular brackets 20 to $25 \mathrm{gm}$. This study was extended 1 year later with the motive force provided by Co-Sm magnets of almost the same volume as previously. The force delivered was increased to 200 'gm wt'. It was concluded that this new magnetic orthodontic tooth movement system was useful not only for space closure, but also for canine retraction and to correct dislocated, rotated or inclined teeth. ${ }^{18}$

\section{Disadvantages}

- Corrosion of magnetic attachments may occur by two different mechanisms as follows:

- Corrosion of the magnet due to the breakdown of the encapsulating material.

- Corrosion of the magnet due to diffusion of moisture and ions through the epoxy seal.

- The main problem associated with the use of magnets as retentive devices is corrosion. Both $\mathrm{Sm}-\mathrm{Co}$ and NdFe-B magnets are extremely brittle and susceptible to corrosion, especially in chloride-containing environments, such as saliva, and the presence of bacteria increases the corrosion of Nd-Fe-B magnets.

- It is, therefore, necessary to encapsulate or coat the magnets for use in dental applications. However, continual wear of the encapsulating material leads to exposure of the magnet.

- The excessive wear of the magnet may be due to the abrasive nature of the titanium nitride-coated soft magnetic tooth keeper that is used with some implant systems.

- Currently, available magnets based on Nd-Fe-B have attractive forces that enable them to provide retention. Problem of corrosion can be overcome with encapsulating materials, such as stainless steel, which are effective.

\section{CONCLUSION}

Early attempts at using magnets for intraoral uses were unsuccessful, mainly because of the large size of magnets at that time and the inadequate forces that they provided. However, since the introduction of rare earth magnets, such as samarium-cobalt and neodymium-iron-boron, it has become possible to produce magnets with small enough dimensions to be used in dental applications and still provide the necessary force. Introduction of rare earth magnets into orthodontics for various therapeutic uses is very recent. Within 10 years, magnetic forces have gained good acceptance in correction of skeletal 
and dental defects. The main advantage with magnets is operator controlled. It eliminates patient cooperation. Conceivable risks of harmful biological effects are negligible with magnets. It is easy to maintain oral hygiene. Compared with other conventional orthodontic methods of force delivery systems magnets are cost effective. Their high cost can overcome by reusing it after sterilization and recycling. These magnets after recycling have not shown much change in their force system. Magnets suffer from tarnish and corrosion. Tarnish and corrosion products are cytotoxic. Tarnish and corrosive nature is prevented by casing them in stainless steel jackets (or) giving parylene coat. Magnets exert continuous forces with less friction, compared to other conventional orthodontic appliances. Teeth movement are bodily in nature and treatment time is shorter. They can be associated along with fixed, removable and functional appliances. Uses of extraoral forces are minimized and anchorage control with them is very precise. Magnets can be used to give predictable forces in either attraction or repelling mode. The orthodontic stimuli provided by the magnetic appliance have reduced the systemic stress reaction seen with conventional orthodontic mechanotherapy. The incidence of periodontal disturbances, root resorption and caries are considerably low and foremost no discomfort.

\section{REFERENCES}

1. Abraham M Blechman. Magnetic forces in orthodontics. Am J Orthod 1978;74:435.

2. Javid N. The use of magnets in a maxillofacial prosthesis. J Pros Dent 1971;25:334-341.

3. Federick DR. A magnetically retained interim maxillary obturator. J Pros Dent 1976;36:671-675.
4. Gillings BR. Magnetic retention for complete and partial overdentures, Part I. J Pros Dent 1981:45:484-491.

5. Sandler PJ. An attractive solution to unerupted teeth. Am J Orthod and Dentofac Orthoped 1991;100:489-493.

6. Darendeliler MA, Freidle JM. Treatment of an impacted canine with magnet. J Clin Orthod 1994;28:639-642.

7. Blechman AM. Magnetic forces systems in orthodontics. Am J Orthod 1985;87:201-210.

8. Springate SD. Micromagnetic retainers-An attractive solution to fixed retention. British Orthod 1991;18:139-141.

9. Lars Bondemark. Biocompatibility of new, clinically used and recycled orthodontic samarium cobalt magnets. Am J Orthod 1994;105:568-574.

10. Muller M. The use of magnets in orthodontics: an alternative means to produce tooth movement. European J Orthod 1984;6:247-253.

11. Vardimon AD. Magnetic vs Mechanical expansion with different force thresholds and points of force application. Am J Orthod 1987;92:455-466.

12. Gianelly AA. Distalization of molars with repelling magnets. J Clin Orthod 1988;22:40-44

13. Vardimon AD. Functional orthopaedic magnetic appliance (FOMA) III. Am J Orthod 1990;97:135-148.

14. Ali Darendeliler. Magnetic Activator Device (MAD-II) for correction of class II Div-I malocclusions. Am J Orthod 1993; 103:223-239.

15. Chate RAC. The propellant unilateral magnetic appliance (PUMA): a new technique for hemifacial microsomia. European J Orthod 1995;17:263-271.

16. Moss JP, Linney AD, Goodwin P, Shaw IA. Three-dimensional study of treatment with magnet and non-magnet twin blocks. European J Orthodont 1993;15:342.

17. Kawata T, Takeda S. A new orthodontic appliance by means of a magnetic bracket. J Dent Res (SPL issue) 1977;56:587.

18. Ichikawa T, Horiuchi M, Wigianto R, Matsumoto N. In vitro study of mandibular implant retained overdentures: the influence of stud attachments on load transfer to the implant and soft tissue. Int J Prosthodont 1996;9:394-399. 